Proceedings of the Institute of Mathematics and Mechanics,

National Academy of Sciences of Azerbaijan

Volume 46, Number 1, 2020, Pages 45-55

https://doi.org/10.29228/proc. 16

\title{
CONFORMAL RICCI SOLITONS OF LAGRANGIAN SUBMANIFOLDS IN KÄHLER MANIFOLDS
}

\author{
MOHD. DANISH SIDDIQI
}

\begin{abstract}
The object of the paper is to study a compact Lagrangian submanifold $M$ in Kähler manifolds, such that the induced metric on the Lagrangian submanifolds is a conformal Ricci soliton with respect to potential vector field given by mean curvature vector field. Moreover, we also discuss the gradient conformal Ricci soliton and prove two characterizations of conformal Ricci soliton with the Laplace operator and the Poisson equation.
\end{abstract}

\section{Introduction}

The study of Lagrangian submanifolds in Kähler manifolds and in the nearly Kähler six-sphere is a very active area during the last quarter of century. Many interesting results on Lagrangian submanifolds have been obtained by many geometers. The Lagrangian submanifolds have been important geometric objects of study in symplectic geometry [7]. In 1993, the problem of minimizing the volume of Lagrangian submanifolds under Hamiltonian deformations was introduced by Y. G. Oh [9]. Hamiltonian minimal submanifolds are interesting and important object among Lagrangian submanifolds [2]. Using Y. G. Oh's [9] result, we can see that compact Lagrangian submanifold $M$ in a Kähler manifold $\bar{M}$ is Hamiltonian manifold if and only if tangent vector field $J H$ is divergence free of $M$, respectively.

On the other hand Ricci flow are intrinsic geometric flows on a pseudo - Riemannian manifold, whose fixed points are solitons, it was introduced by R. S. Hamilton [5]. In [4], A.E. Fischer introduced a new concept called conformal Ricci flow which is a variation of the classical Ricci flow equation that modifies the unit volume constraint of that equation to a scalar curvature constraint. Since the conformal geometry plays an important role to constrain the scalar curvature and the equations are the vector field sum of a conformal flow equation and a Ricci flow equation, the resulting equations are named as the conformal Ricci flow equations. These new equations are given by

$$
\frac{\partial g}{\partial t}=-2 S-\left(p+\frac{2}{n}\right) g
$$

2010 Mathematics Subject Classification. 53C15, 53C20, 53C25, 53C44.

Key words and phrases. Conformal Ricci soliton, Kähler manifolds, Lagrangian submanifolds, Laplace operator, Poisson equation. 
where $r(g)=-1$ and $p$ is a non-dynamical scalar field (time dependent scalar field), $r(g)$ is the scalar curvature of the manifold and $n$ is the dimension of the manifold $M$.

The conformal Ricci flow equations are analogous to the Navier-Stokes equations of fluid mechanics and because of this analogy the time dependent scalar field $p$ is called a conformal pressure and, as for the real physical pressure in fluid mechanics that serves to maintain the in-compressibility of the fluid, the conformal pressure serves as a Lagrange multiplier to conformally deform the metric flow so as to maintain the scalar curvature constraint. The equilibrium points of the conformal Ricci flow equations are Einstein metrics with Einstein constant $\frac{-1}{n}$. Thus the conformal pressure $p$ is zero at an equilibrium point and positive otherwise. In 2015, N. Basu and A. Bhattacharyya [1] introduced the notion of conformal Ricci soliton and the equation is as follows

$$
\mathcal{L}_{V} g+2 S+\left[2 \lambda-\left(p+\frac{2}{n}\right)\right] g=0,
$$

where $\lambda$ is a constant. conformal Ricci soliton is said to be shrinking, steady or expanding according as $\lambda<0, \lambda=0$ and $\lambda>0$, respectively. Some extended properties of conformal Ricci solitons was studied by M. D. Siddiqi in [13]. If the vector field $V$ is the gradient of a potential function $f$, then $g$ is called a gradient conformal Ricci soliton. In this paper, we investigate Lagrangian submanifolds, whose induced metric is a conformal Ricci soliton with potential vector field $J H$, in Kähler manifolds and in particular in complex space forms.

\section{Conformal Ricci solitons}

On Riemannian manifold $(M, g)$, from $(1.2)$ we have

$$
\frac{1}{2} \mathcal{L}_{V} g+S+\frac{1}{2}\left[2 \lambda-\left(p+\frac{2}{n}\right)\right] g=0,
$$

where $S$ is the Ricci tensor, and $\mathcal{L}_{V} g$ is the Lie derivative of potential vector field $V$ is define as

$$
\left(\mathcal{L}_{V} g\right)(X, Y)=g\left(\nabla_{X} V, Y\right)+g\left(X, \nabla_{Y} V\right)
$$

where $\nabla$ denotes the Levi-Civita connection of $M$. Hence if $(M, g)$ is a conformal Ricci soliton with potential vector field $V$, then (2.1) and (2.2) imply

$$
\operatorname{div} V+r+\left[\lambda-\frac{1}{2}\left(p+\frac{2}{n}\right)\right] n=0,
$$

where $\operatorname{div} V=\operatorname{trace}\left(X \longmapsto \nabla_{X} V\right)$ is the divergence of $V$ and $r$ is the scalar curvature of $M$. By (2.3) and Green's Theorem, we have

$$
\int_{M} \operatorname{div} V \mu=0
$$

for a compact oriented Riemannian manifold $M$ and $\mu$ is the volume form of $(M, g)$. We obtain

Proposition 2.1. Let $(M, g)$ be a compact oriented conformal Ricci soliton satisfying (1.2) with respect to the potential vector field $V$ on $M$. Then 
(1) (i) $\int_{M} r \mu=-\left[\lambda-\frac{1}{2}\left(p+\frac{2}{n}\right)\right] n \operatorname{Vol}(M)$.

(2) (ii) The scalar curvature $r$ of $M$ is constant if and only if $\operatorname{div} V=0$.

(3) (iii) If either $r \leq-\left[\lambda-\frac{1}{2}\left(p+\frac{2}{n}\right)\right] n$ or $r \geq-\left[\lambda-\frac{1}{2}\left(p+\frac{2}{n}\right)\right] n$ on $M$, then $r=-\left[\lambda-\frac{1}{2}\left(p+\frac{2}{n}\right)\right] n$ and $\operatorname{div} V=0$.

Now, we recall the following results due to Hamilton and Ivey (see [6], [5], [3]):

Theorem 2.1. ( $[10],[12])$ Let $(M, g)$ is either 2 or 3 -dimensional compact conformal Ricci soliton with potential vector field $V$. Then sectional curvature of $M$ are constant.

Also, the following is known:

Theorem 2.2. On compact conformal Ricci soliton $(M, g)$, if the scalar curvature is constant, then $(M, g)$ is Einstein.

\section{Lagrangian submanifolds}

In [9] Y. G. Oh defined Hamiltonian deformation of Lagrangian submanifolds in Kähler manifold. Let $\bar{M}$ be a complex $n$-dimensional Kähler manifold with Kähler form $\omega$, Riemannian metric $\langle$,$\rangle and complex structure J$. Let $\pi: M \longrightarrow \bar{M}$ be a Lagrangian immersion from a real $n$-dimensional manifold $M$ to $\bar{M}$, i.e., $\left.\omega\right|_{T M}=$ 0 . For a vector field $V$ along $\pi$, we define a 1-form $\alpha_{V}$ on $M$ as $\alpha_{V}=\left.g(J V,)\right|_{T M$.$} .$ Smooth family embeddings $e_{d}: M \longrightarrow P$ is called Hamiltonian deformation if for variational vector field $V$, the 1 -form $\alpha_{V}$ is exact. A Lagrangian submanifold $M$ is Hamiltonian minimal (or $H$-minimal) if $M$ is stationary for any Hamiltonian deformation. Y.G. Oh [9] showed that when $M$ is compact, $M$ is $H$-minimal if and only if $\alpha_{H}$ is co-closed, i.e., Euler-Lagrange's equation- $\delta \alpha_{H}=0$ where $H$ is the mean curvature vector field of $M$. We have

$$
M \quad \text { is Hamiltonian minimal } \Leftrightarrow \operatorname{div} J H=0 .
$$

For, Lagrangian submanifold $M$ in a Kähler manifold $\bar{M}$ and the induced metric $g$ on $M$, the following relations hold:

$$
\nabla h=0 \Rightarrow \nabla^{\perp} H=0 \Rightarrow \mathcal{L}_{J H g}=0 \Rightarrow \operatorname{div} J H=0,
$$

where $h$ and $\nabla^{\perp}$ denote second fundamental form and normal connection of $M$ in $\bar{M}$ respectively, and $\nabla h$ is defined as

$$
\left(\nabla_{X} h\right)(Y, Z)=\nabla_{X}^{\perp} h(Y, Z)-h\left(\nabla_{X} Y, Z\right)-h\left(Y, \nabla_{X} Z\right)
$$

for tangent vector fields $X, Y, Z$ on $M$. We note that

$$
\nabla_{X}(J H)=J \nabla^{\perp} H .
$$

Let $\bar{M}^{n}(4 c)$ be an $n$-dimensional complex space from with constant holomorphic sectional curvature $4 c$ and let $M=M^{n}$ be a Lagrangian submanifold in $\bar{M}^{n}(4 c)$.

The Gauss equation is

$$
R(X, Y) Z=c\{g(Y, Z) X-g(X, Z) Y\}+A_{h(Y, Z)} X-A_{h(X, Z)} Y,
$$


where $R$ and $A$ denote curvature tensor and shape operator of $M$ with relation

$$
\langle(h(X, Y), \xi)\rangle=g\left(A_{\xi} X, Y\right)
$$

for tangent vector fields $X, Y$ and normal vector field $\xi$ on $M$, respectively. The Ricci tensor $M$ is given by[3]

$$
S(Y, Z)=(n-1) c g(Y, Z)+\langle(h(Y, Z), H)\rangle-\operatorname{trace}_{g}\left(X \longmapsto A_{h(X, Z)} T\right) .
$$

The sclar curvature $r$ of $M$ is

$$
r=n(n-1) c+\|H\|^{2}-\|h\|^{2} .
$$

Let $T$ be a symmetric $(0,3)$-tensor field on $M$ defined by

$$
T(X, Y, Z)=\langle h(X, Y), J Z\rangle .
$$

Then Codazzi equation

$$
\left(\nabla_{X} h\right)(Y, Z)=\left(\nabla_{Y} h\right)(X, Z)
$$

implies that $\nabla T$ is a symmetric $(0,4)$-tensor field on $M$. Combining with (3.2), we can easily obtain followings theorem:

Theorem 3.1. Let $M$ be a Lagrangian submanifold in a complex space form of constant holomorphic sectional curvature. Then the mean curvature vector field $H$ is parallel with respect to the normal connection $\nabla^{\perp}$ if and only if $J H$ is a Killing vector field on $M$.

\section{Conformal Ricci soliton of Lagrangian submanifolds}

Let $M=M^{n}$ be a Lagrangian submanifolds in a Kähler manifold $\bar{M}^{n}$, and let $g$ be the induced metric on $M$. Suppose $(M, g)$ is a conformal Ricci soliton (1.2) with $V=J H$. By Proposition (2.1), Theorem (3.1) and also from equations (2.4) and (3.1), we obtain the following:

Theorem 4.1. Let $M^{n}$ be a compact oriented Lagrangian submanifold in a Kähler manifold $\bar{M}^{n}$ and let $g$ be the induced metric on $M$. Suppose $(M, g)$ is a conformal Ricci soliton with $V=J H$. Then we have

(1) (i) If $M$ is Hamiltonian minimal, then $M$ is Einstein manifold and $J H$ is a Killing vector field on $M$.

(2) (ii) If the scalar curvature $r$ of $(M, g)$ satisfies either $r \leq-\left[\lambda-\frac{1}{2}\left(p+\frac{2}{n}\right)\right] n$ or $r \geq-\left[\lambda-\frac{1}{2}\left(p+\frac{2}{n}\right)\right] n$, then $r=-\left[\lambda-\frac{1}{2}\left(p+\frac{2}{n}\right)\right] n$ and $M$ is Einstein and the same conclusion as (i) holds.

We note that Lagrangian submanifolds $M^{n}$ with parallel second fundamental form in complex space forms are classified (see [2], [10], [12]). By (3.1) and (3.2), they are Hamiltonian minimal. Also they are locally symmetric and for irreducible once, they are Einstein. Hence irreducible Lagrangian submanifolds in complex space forms satisfying $\nabla h=0$ are considered as conformal Ricci solitons with Killing potential vector field $J H$. When $\operatorname{dim} M=2$ or 3 , by Theorem (2.2) and equations (3.1), (3.2), we have 
Theorem 4.2. Let $M$ be a compact Lagrangian submanifold in a Kähler manifold $\bar{M}$ with $\operatorname{dim} M=2$ or 3 , and let $g$ be the induced metric on $M$. Suppose $(M, g)$ is a conformal Ricci soliton with $V=J H$. Then $(M, g)$ is constant curvature and $\nabla^{\perp} H=0$.

Let $\bar{M}^{n}(4 c)$ be an $n$-dimensional complex space form with constant holomorphic sectional curvature $4 c$. For Lagrangian submanifold $(M, g)$ in $\bar{M}^{n}(4 c)$, using (2.2) and (3.3) we obtain that conformal Ricci soliton equation (1.2) with $V=J H$ is written as

$$
\begin{gathered}
\frac{1}{2}\left[g\left(\nabla_{X}(J H), Y\right)+g\left(X, \nabla_{Y}(J H)\right)\right]-\langle h(X, Y), H\rangle \\
+\operatorname{trace}\left(Z \longmapsto A_{h(Z, Y)} X\right)+\left((n-1) c+\left[\lambda-\frac{1}{2}\left(p+\frac{2}{n}\right)\right]\right) g(X, Y)=0 .
\end{gathered}
$$

After contracting with respect to $X$ and $Y$, we obtain

$$
\operatorname{div} J H-\|H\|^{2}+\|h\|^{2}+n\left((n-1) c+\left[\lambda-\frac{1}{2}\left(p+\frac{2}{n}\right)\right]\right)=0,
$$

which is clearly equivalent to (2.4) with $V=J H$ by (3.4). Now, taking covariant differentiation of (4.1) by $W$, we get

$$
\begin{aligned}
\frac{1}{2} g\left(\nabla_{W} \nabla_{X}(J H)\right. & \left.-\nabla_{\nabla_{W} X}(J H), Y\right)+\frac{1}{2} g\left(X, \nabla_{W} \nabla_{Y}(J H)-\nabla_{\nabla_{W} Y}(J H)\right) \\
& -\left\langle\left\langle\nabla_{W} h(X, Y), H\right\rangle\right\rangle-\left\langle h(X, Y), \nabla_{W}^{\perp} H\right\rangle \\
+\operatorname{trace}(Z \longmapsto & \left.\left(\nabla_{W} A\right)_{h(Z, Y)} X\right)+\operatorname{trace}\left(Z \longmapsto A_{\left(\nabla_{W} h\right)(Z, Y)}, X\right)=0 .
\end{aligned}
$$

Then Codazzi equation (3.5) yields that

$$
\begin{gathered}
\frac{1}{2} g(R(W, X) J H, Y)+\frac{1}{2} g\left(X, \nabla_{W} \nabla_{Y}(J H)-\nabla_{\nabla_{W} Y}(J H)\right) \\
-\frac{1}{2} g\left(W, \nabla_{X} \nabla_{Y}(J H)-\nabla_{\nabla_{X} Y}(J H)\right)-\left\langle h(X, Y), \nabla_{W}^{\perp} H\right\rangle+\left\langle h(W, Y), \nabla_{X}^{\perp} H\right\rangle \\
+\operatorname{trace}\left(Z \longmapsto\left(\nabla_{W} A\right)_{h(Z, Y)} X\right)+\operatorname{trace}\left(Z \longmapsto A_{\left(\nabla_{W} h\right)(Z, Y)}, X\right)=0 . \\
-\operatorname{trace}\left(Z \longmapsto\left(\nabla_{X} A\right)_{h(Z, Y)} W\right)-\operatorname{trace}\left(Z \longmapsto A_{\left(\nabla_{X} h\right)(Z, Y)}, W\right)=0 .
\end{gathered}
$$

By contracting with respect to $W$ and $Y$, and using (3.5) and (4.2) we get

$$
S(X, J H)+g(X, \Delta(J H))=0 .
$$

Because of

and

$$
\begin{aligned}
\frac{1}{2} \Delta\|H\|^{2}= & \frac{1}{2} \Delta\|J H\|^{2}=g(\Delta(J H), J H)+\|\nabla(J H)\|^{2} \\
& =-S(J H, J H)+\left\|\nabla^{\perp} H\right\|^{2}
\end{aligned}
$$

$$
\int_{M} \Delta\|H\|^{2} \mu=0
$$

when $M$ is compact and oriented, the following results holds: 
Theorem 4.3. Let $M^{n}$ be a compact oriented Lagrangian submanifold in a complex space form $\bar{M}^{n}(4 c)$, and let $g$ be the induced metric on $M$. Suppose $(M, g)$ is a conformal Ricci soliton with $V=J H$. If $S(J H, J H) \leq 0$ on $M$, then $\nabla^{\perp} H=0$ and $(M, g)$ is conformal Ricci flat.

With respect to either 2 or 3-dimensional Lagrangian submanifold in complex space forms, Theorem (4.2) is reduce to:

Theorem 4.4. Let $M^{n}$ be a compact Lagrangian submanifold in a complex space form $\bar{M}^{n}(4 c)$ with $n=2,3$ and let $g$ be the induced metric on $M$. Suppose $(M, g)$ is a conformal Ricci soliton with $V=J H$. Then $(M, g)$ is either totally geodesic or flat with parallel mean curvature vector field.

\section{Gradient conformal Ricci soliton}

It is a well known fact that if $\left(M, g, \xi,\left[\lambda-\frac{1}{2}\left(p+\frac{2}{n}\right)\right]\right)$ is a compact conformal Ricci soliton, then the potential field $\xi$ is a gradient of some smooth function $\psi$ up to the addition of a Killing vector field and thus a compact conformal Ricci soliton is a gradient conformal Ricci soliton [11]. We shall denote a gradient conformal Ricci soliton by $\left(M, g, \psi,\left[\lambda-\frac{1}{2}\left(p+\frac{2}{n}\right)\right]\right)$ and called the smooth function $\psi$ the potential function of the gradient conformal Ricci soliton $\left(M, g, \psi,\left[\lambda-\frac{1}{2}\left(p+\frac{2}{n}\right)\right]\right)$ it is always possible to choose the potential function $\psi$ satisfying

$$
r+\|\nabla \psi\|^{2}=2\left[\lambda-\frac{1}{2}\left(p+\frac{2}{n}\right)\right] \psi,
$$

where $r$ denotes the scalar curvature of $M$ [12]. A gradient conformal Ricci soliton $\left(M, g, \psi,\left[\lambda-\frac{1}{2}\left(p+\frac{2}{n}\right)\right]\right)$ with such a potential function is simply called a gradient conformal Ricci soliton with normalized potential [8].

On a compact Riemannian manifold $(M, g)$ and a smooth function $\phi: M \longrightarrow$ $\mathbb{R}$, the average value of $\phi$, denotes by $\phi_{a v}$, is a real number defined by [14]

$$
\phi_{a v}=\frac{1}{\operatorname{Vol}(M)} \int_{M} \phi .
$$

Let $\left(M, g, \psi,\left[\lambda-\frac{1}{2}\left(p+\frac{2}{n}\right)\right]\right)$ be an $n$-dimensional gradient conformal Ricci soliton. Then we have

$$
H e s s \psi+S=\left[\lambda-\frac{1}{2}\left(p+\frac{2}{n}\right)\right] g,
$$

$X, Y \in \chi(M)$, where $\operatorname{Hess} \psi(X, Y)=g\left(\nabla_{X} \nabla_{\psi}, Y\right)$ is the Hessian and $\nabla \psi$ is the gradient of the potential function $\psi$.

Since $r$ is the scalar curvature of $(M, g)$, equation (5.3) yields

$$
\Delta \psi=n\left[\lambda-\frac{1}{2}\left(p+\frac{2}{n}\right)\right]-r,
$$

where $\Delta \psi=\operatorname{Trace}($ Hess $\psi)$ is the Laplacian of $\psi$. The Ricci operator $Q$ is defined by

$$
S(X, Y)=g(Q X, Y), \quad X, Y \in \chi(M) .
$$


It is well known that the Ricci operator $Q$ satisfies

$$
\sum(\nabla Q)\left(e_{i}, e_{i}\right)=\frac{1}{2} \nabla r,
$$

where $\left\{e_{i}, \ldots . . e_{n}\right\}$ is a local orthonormal frame and $\nabla Q$ is the covariant derivative of $Q$ defined by

$$
(\nabla Q)(X, Y)=\nabla_{X}(Q Y)-Q\left(\nabla_{X} Y\right)
$$

We define the symmetric operator $A_{\psi}$ by

$$
H \operatorname{Hess} \psi(X, Y)=g\left(A_{\psi} X, Y\right), \quad X, Y \in \chi(M) .
$$

Then by using the definition of curvature tensor $R$, we have

$$
\left(\nabla A_{\psi}\right)(X, Y)-\left(\nabla A_{\psi}\right)(Y, X)=R(X, Y) \nabla \psi
$$

After applying the above equation $\Delta \psi=\operatorname{Trace}\left(A_{\psi}\right)$, and the symmetry of $A_{\psi}$, we obtain

$$
\begin{gathered}
X(\Delta \psi)=\sum_{i} g\left(\left(\nabla A_{\psi}\right)\left(X, e_{i}\right), e_{i}\right) \\
=\sum_{i} g\left(\left(\nabla A_{\psi}\right)\left(X, e_{i}\right)+R\left(X, e_{i}\right) \nabla \psi, e_{i}\right) \\
-S(X, \nabla \psi)+\sum_{i} g\left(\left(\nabla A_{\psi}\right)\left(e_{i}, e_{i}\right), X\right)
\end{gathered}
$$

for $X \in \chi(M)$. Also, it follows from equation (5.3) that

$$
\left(\nabla A_{\psi}\right)(X, Y)=-(\nabla Q)(X, Y) .
$$

By substituting this equation (5.7) and using (5.4) and (6), we find

$$
-X(r)=-S(X, \nabla \psi)-\frac{1}{2} X(r)
$$

which implies

$$
Q(\nabla \psi)=\frac{1}{2} \nabla r
$$

Note that on a connected gradient conformal Ricci soliton $\left(M, g, \psi,\left[\lambda-\frac{1}{2}\left(p+\frac{2}{n}\right)\right]\right)$, it follows from equations (5.3) and (5.8) that

$$
\frac{1}{2} X\left(\|\nabla \psi\|^{2}+r\right)=\operatorname{Hess} \psi(X, \nabla \psi)+S(X, \nabla \psi)=\left[\lambda-\frac{1}{2}\left(p+\frac{2}{n}\right)\right] g(X, \nabla \psi),
$$

that is

This shows that

$$
\left.X\left(\|\nabla \psi\|^{2}+r-2\left[\lambda-\frac{1}{2}\left(p+\frac{2}{n}\right)\right]\right) \psi\right)=0 .
$$

$$
\frac{1}{2}\left(\|\nabla \psi\|^{2}+r-\left[\lambda-\frac{1}{2}\left(p+\frac{2}{n}\right)\right]\right) \psi=k
$$

for a constant $k$. Now, after replacing the potential function $\psi$ of the connected shrinking gradient conformal Ricci soliton $\left(M, g, \psi,\left[\lambda-\frac{1}{2}\left(p+\frac{2}{n}\right)\right]\right)$ by $\psi-\frac{k}{\left[\lambda-\frac{1}{2}\left(p+\frac{2}{n}\right)\right]}$, we see that the shrinking gradient conformal Ricci soliton satisfies

$$
2\left[\lambda-\frac{1}{2}\left(p+\frac{2}{n}\right)\right] \psi=\|\nabla \psi\|^{2}+r .
$$


On a gradient conformal Ricci soliton, equation (5.4) gives

$$
\int_{M}\left(n\left[\lambda-\frac{1}{2}\left(p+\frac{2}{n}\right)\right]-r\right)=0 \text {. }
$$

Now, we can prove the following characterization of trivial conformal Ricci solitons:

Theorem 5.1. An n-dimensional shrinking gradient conformal Ricci soliton $\left(M, g, \psi,\left[\lambda-\frac{1}{2}\left(p+\frac{2}{n}\right)\right]\right)$ with normalized potential is trivial if and only if

$$
(\psi r)_{a v} \leq \frac{1}{2} n^{2}\left[\lambda-\frac{1}{2}\left(p+\frac{2}{n}\right)\right]
$$

where $r$ denotes the scalar curvature of $M$.

Proof. Let $\left(M, g, \psi,\left[\lambda-\frac{1}{2}\left(p+\frac{2}{n}\right)\right]\right)$ be an $n$-dimensional shrinking gradient conformal Ricci soliton. Then, it follows from (5.4) and (5.9) that

$$
\begin{gathered}
\frac{1}{2} \Delta \psi^{2}=\psi \Delta \psi+\|\nabla \psi\|^{2} \\
=(n+2)\left[\lambda-\frac{1}{2}\left(p+\frac{2}{n}\right)\right] \psi-\psi r-r,
\end{gathered}
$$

which together with equation (5.10) gives

$$
\int_{M} \psi r=\left[\lambda-\frac{1}{2}\left(p+\frac{2}{n}\right)\right](n+2) \int_{M}\left(\psi-\frac{n}{n+2}\right) .
$$

Note that equations (5.9) and (5.10) imply

$$
\int_{M}\left(\psi-\frac{n}{2}\right)=\frac{1}{2\left[\lambda-\frac{1}{2}\left(p+\frac{2}{n}\right)\right]} \int_{M}\|\nabla \psi\|^{2}
$$

which together with equation (5.12) gives

$$
\int_{M} \psi r=\frac{1}{2} n^{2}\left[\lambda-\frac{1}{2}\left(p+\frac{2}{n}\right)\right] \operatorname{Vol}(M)+\frac{n+2}{2} \int_{M}\|\nabla \psi\|^{2} .
$$

If the condition $(\psi r)_{a v} \leq \frac{1}{2} n^{2}\left[\lambda-\frac{1}{2}\left(p+\frac{2}{n}\right)\right]$ holds, then we shall have

$$
\int_{M} \psi r=\frac{1}{2} n^{2}\left[\lambda-\frac{1}{2}\left(p+\frac{2}{n}\right)\right] \operatorname{Vol}(M) .
$$

By combining (5.14) and (5.15), we obtain

$$
\int_{M}\|\nabla \psi\|^{2}=0
$$

which implies that the potential function $\psi$ is a constant. Consequently, it follows from (refs4) that $M$ is an Einstein manifold. Thus The conformal Ricci soliton is trivial.

Conversely, if an $n$-dimensional compact shrinking gradient conformal Ricci soliton is trivial, then $r=n\left[\lambda-\frac{1}{2}\left(p+\frac{2}{n}\right)\right]$ and $\psi$ is constant. Therefore, by the equation (5.9) we obtain $\psi=\frac{2}{2(\psi r)_{a v} \leq \frac{1}{2} n^{2}\left[\lambda-\frac{1}{2}\left(p+\frac{2}{n}\right)\right]}$. Consequently, we have $(\psi r)_{a v}=\frac{1}{2} n^{2}\left[\lambda-\frac{1}{2}\left(p+\frac{2}{n}\right)\right]$. This complete the proof. 


\section{Poisson equation}

The Poisson equation on a Riemannian manifold $(M, g)$ is

$$
\Delta \varphi=\sigma,
$$

where $\Delta$ is the Laplace operator, $\sigma$ is the given function, and $\varphi$ is the solution to be determined. The Poisson equation plays an fundamental role in Physics, also well known for its importance in Electrostatics, Biophysics and Engineering.

It is known that the Poisson equation $\Delta \varphi=\sigma$ on a compact Riemannian manifold $(M, g)$ has a unique solution up constants if and only if the integral of $\sigma$ is equal to zero [12]. Also, in order to use a Poisson equation to study the geometry of gradient conformal Ricci soliton $\left(M, g, \psi,\left[\lambda-\frac{1}{2}\left(p+\frac{2}{n}\right)\right]\right)$, we need to construct a function $\sigma$ whose integral is equal to zero.

On a gradient conformal Ricci soliton $\left(M, g, \psi,\left[\lambda-\frac{1}{2}\left(p+\frac{2}{n}\right)\right]\right)$ the function $\sigma=$ $\lambda_{1}\left(n \lambda_{1}-r\right)$ where $\left(\lambda_{1}=\left[\lambda-\frac{1}{2}\left(p+\frac{2}{n}\right)\right]\right)$ satisfies the property $\int_{M} \sigma=0$. We study the Poisson equation $\Delta \varphi=\sigma$ with $\sigma=\lambda_{1}\left(n \lambda_{1}-r\right)$ on the gradient conformal Ricci soliton .

Now, we have the following theorem:

Theorem 6.1. Let $\left(M, g, \psi, \lambda_{1}\right)$ be an $n$-dimensional shrinking gradient conformal Ricci soliton and let $\sigma=\lambda_{1}\left(n \lambda_{1}-r\right)$. If the scalar curvature $r$ is a solution of the Poisson equation

$$
\Delta \varphi=\sigma,
$$

then either $M$ is trivial or the first nonzero eigenvalue $\lambda_{L}$ of the Laplace operator $\Delta$ of $M$ satisfies $\lambda_{L} \leq \lambda_{1}$.

Proof. Let $\left(M, g, \psi, \lambda_{1}\right)$ be an $n$-dimensional shrinking gradient conformal Ricci soliton [11] and the scalar curvature $r$ satisfies the Poisson equation (see [8], [10])

$$
\Delta \varphi=\sigma,
$$

with $\sigma=\lambda_{1}\left(n \lambda_{1}-r\right)$. Note that the function $\omega=\frac{1}{2}\left(\|\nabla \psi\|^{2}+r\right)$ satisfies

$$
\omega=\lambda_{1} \psi
$$

due to equation (5.9). Combining this with equation (5.5) gives

$$
\Delta \omega=\lambda_{1}\left(n \lambda_{1}-r\right)=\sigma .
$$

Therefore, both $r$ and $\omega$ are the solutions of the Poisson equation (6.2). Hence, we have $r=\omega+k$ for some constant $k$. Consequently (see [8], [10]), we obtain

$$
\int_{M}\|\nabla r\|^{2} \geq \lambda_{L} \int_{M}\left(n \lambda_{1}-r\right)^{2} .
$$

On the other hand, it follows from (5.10) that

$$
\int_{M}\left(n \lambda_{1}-r\right)^{2}=\int_{M}\left(r^{2}-n^{2} \lambda_{1}^{2}\right) .
$$

Consequently, inequality (6.6) takes the form

$$
\int_{M}\|\nabla r\|^{2} \geq \lambda_{L} \int_{M}\left(r^{2}-n^{2} \lambda_{1}^{2}\right)
$$


Because the scalar curvature $r$ is a solution of the Poisson equation (6.2) with $\sigma=\lambda_{1}\left(n \lambda_{1}-r\right)$. we have

$$
r \Delta r=\lambda_{1}\left(n \lambda_{1} r-r^{2}\right)
$$

By applying integration by parts to the last equation and by using equation (5.10), we obtain

$$
\int_{M}\|\nabla r\|^{2} \geq \lambda_{1} \int_{M}\left(r^{2}-n^{2} \lambda_{1}^{2}\right)
$$

which together with using the inequality (6.8) gives

$$
\left(\lambda_{L}-\lambda_{1}\right) \int_{M}\left(r^{2}-n^{2} \lambda_{1}^{2}\right) \geq 0
$$

Now using (5.5), we have

$$
n^{2} \lambda_{1}^{2}-r^{2}=\left(n \lambda_{1}+r\right) \Delta \psi=n \lambda_{1} \Delta \psi+r \Delta \psi,
$$

which on insertion in the above inequality gives

$$
\left(\lambda_{L}-\lambda_{1}\right) \int_{M}(r \Delta \psi) \geq 0
$$

From (5.5), (6.11), $\Delta r=\lambda_{1}\left(n \lambda_{1}-r\right)$ and integration by parts, we get

$$
\begin{aligned}
0 \leq & \left(\lambda_{L}-\lambda_{1}\right) \int_{M} r\left(n \lambda_{1}-r\right) . \\
& =\left(\lambda_{L}-\lambda_{1}\right) \int_{M}(r \Delta \psi) \\
& =\frac{\left(\lambda_{L}-\lambda_{1}\right)}{\lambda_{1}} \int_{M}(r \Delta r) . \\
& =\frac{\left(\lambda_{L}-\lambda_{1}\right)}{\lambda_{1}} \int_{M}\|\nabla r\|^{2}
\end{aligned}
$$

By combining this with (6.4), we obtain

$$
=\left(\lambda_{L}-\lambda_{1}\right) \lambda_{1} \int_{M}\|\nabla \psi\|^{2} \leq 0
$$

which implies that either $\lambda_{L} \leq \lambda_{1}$ holds or $\left(M, g, \psi, \lambda_{1}\right)$ is trivial. This completes the proof.

Acknowledgment. The author expresses his gratitude to the referee for his/her valuable comments and suggestions towards the improvement of the paper. 


\section{References}

[1] N. Basu, A. Bhattacharyya, Conformal Ricci soliton in Kenmotsu manifold, Global Journal of Advanced Research on Classical and Modern Geometries, 4 (2015), 15-21.

[2] B. Y. Chen, Classification of Lagrangian surfaces of constant curvature in the complex Euclidean plane, Proc. Edinb. Math. Soc., 48 (2005), 337-364.

[3] J. T. Cho, M. Kimura, Ricci solitons and Lagrangian subamnifolds in Kähler manifolds, Mem. Fac. Sci. Engg. Shimane. Univ. Series B, Math. Sci., 43 (2010), 27-32.

[4] A. F. Fischer, An introduction to conformal Ricci flow, Classical Quantum Grav., 21(2004) S171-S218.

[5] R. S. Hamilton, The Ricci flow on surfaces, Contemporary Mathematics, 71 (1988), 237-261.

[6] T. Ivey, Ricci solitons of compact three-manifolds, Differ. Geom. Appl. 3 (1993), 301-307.

[7] M. Kimura, Lagrangian submanifolds with codimension 1 totally geodesic foliation in complex projective space, Kodai Math. J, 31 (2008), 38-45.

[8] J. Lott, The work of Grigory Perelman, International Congress of Mathemati-cians, Vol.I, Eur. Math. Soc., Zurich, (2007), 66-76.

[9] Y. G. Oh, Volume minimization of Lagrangian submanifolds under Hamiltonian deformations, Math. Z, 212 (1993), 175-192.

[10] T. Ogata, Surfaces with parallel mean curvature vector in $P^{2}(C)$, Kodai Math. J., 18 (1995), 397-407.

[11] P. Petersen and W. Wylie, On Gradient Ricci solitons with symmetry, Proc. Amer. Math. Soc., 137 (2009), 2085-2092.

[12] G. Perelman, The entropy formula for the Ricci flow and its geometric applications, Preprint, 2002, Zbl 1130.53001; arXiv math/0211159.

[13] M . D. Siddiqi, Conformal $\eta$-Ricci solitons in $\delta$-Lorentzian Trans Sasakian manifolds, Int. J. Maps Math. (IJMM.), 1(2018) 15-34.

[14] K. Yano, Integral formulas in Riemannian geometry, Marcel Dekker, New York, 1970.

Mohd. Danish Siddiqi

Department of Mathematics, Faculty of science, Jazan University, P. Box 114, Jazan, Kingdom of Saudi Arabia,

E-mail address: anallintegral@gmail.com, msiddiqi@jazanu.edu.sa

Received: April 19, 2019; Accepted: November 30, 2019 